\title{
The Distribution and Quantiles of Functionals of Weighted Empirical Distributions when Observations have Different Distributions
}

by

\author{
Christopher S. Withers \\ Applied Mathematics Group \\ Industrial Research Limited \\ Lower Hutt, NEW ZEALAND \\ Saralees Nadarajah \\ School of Mathematics \\ University of Manchester \\ Manchester M13 9PL, UK
}

\begin{abstract}
This paper extends Edgeworth-Cornish-Fisher expansions for the distribution and quantiles of nonparametric estimates in two ways. Firstly it allows observations to have different distributions. Secondly it allows the observations to be weighted in a predetermined way. The use of weighted estimates has a long history including applications to regression, rank statistics and Bayes theory. However asymptotic results have generally been only first order (the CLT and weak convergence). We give third order asymptotics for the distribution and percentiles of any smooth functional of a weighted empirical distribution, thus allowing a considerable increase in accuracy over earlier CLT results.

Consider independent non-identically distributed (non-iid) observations $X_{1 n}, \ldots, X_{n n}$ in $R^{s}$. Let $\widehat{F}(x)$ be their weighted empirical distribution with weights $w_{1 n}, \ldots, w_{n n}$. We obtain cumulant expansions and hence Edgeworth-Cornish-Fisher expansions for $T(\widehat{F})$ for any smooth functional $T(\cdot)$ by extending the concepts of von Mises derivatives to signed measures of total measure 1. As an example we give the cumulant coefficients needed for Edgeworth-Cornish-Fisher expansions to $O\left(n^{-3 / 2}\right)$ for the sample variance when observations are non-iid.
\end{abstract}

Keywords: Edgeworth-Cornish-Fisher expansions; von Mises derivatives; Weighted empirical distribution.

\section{Introduction and Summary}

Withers $(1983,1988)$ gave third order asymptotics for the distribution of functionals of empirical (or sample) distributions for iid observations. This paper extends these results to non-iid weighted observations.

Traditional inference is based on the empirical distribution function. This gives each observation equal weight. However in many contexts it is more appropriate to weight the observations differently. An important class of weighted statistics are the rank statistics studied by Hajek and Sidak (1967). They gave first order (asymptotic) results both for 
iid observations, and for the contiguous case where the observations are from distributions approaching the null case. However they did not deal with the case where observations have fixed distinct distributions. A seminal contribution to the theory of weighted empirical distributions was made by Koul (1992) who gave first order properties for linear models. This was extended by another seminal contribution, Koul (2002), to allow for random weights with applications to $M$ - and $R$ - estimates as well as to autoregressive processes. However, he confined his focus to first order (weak convergence) results. Optimality of certain weights in a Bayesian setting was proved by Chernoff and Zacks (1964) for testing the hypothesis of a jump in the mean. For other work, including a comprehensive account of the literature, we refer the readers to Lahiri (1992a, 1992b, 1992c) and Lahiri (2003).

This paper - following on from Withers and Nadarajah (2008) - gives cumulant expansions - and hence Edgeworth-Cornish-Fisher expansions - for smooth functionals of weighted empirical distributions for arbitrary non-iid observations. These cumulant expansions are given in Section 2. As simple examples, Section 3 applies these results to the mean and variance. Section 4 gives a chain rule for the functional derivative of a function of several functionals, and uses this to obtain the leading cumulant coefficients for the Studentized mean, and the coefficient of variation. For completeness the Edgeworth-Cornish-Fisher expansions for the distribution and quantiles are given in Appendix A, as well as the multivariate Edgeworth expansion.

Let $X_{1 n}, \ldots, X_{n n}$ be independent random variables in $R^{s}$ with distributions $F_{1 n}, \ldots, F_{n n}$. Let $w_{1 n}, \ldots, w_{n n}$ be given real numbers adding to $n$ :

$$
w_{1 n}+\ldots+w_{n n}=n
$$

The simplest example giving more weight to the later observations are the weights $w_{i n}=$ $2 i /(n+1)$ shown by Chernoff and Zacks (1964) to be optimal in a Bayesian setting for testing for a jump in the mean. The mean with these weights was also used by Kander and Zacks (1966) and others.

Define the weighted empirical distribution as

$$
\widehat{F}(x)=n^{-1} \sum_{i=1}^{n} w_{i n} I\left(X_{i n} \leq x\right)
$$

for $x$ in $R^{s}$, where $I(A)=1$ or 0 for $A$ true or false. Its mean is

$$
F(x)=E \widehat{F}(x)=n^{-1} \sum_{i=1}^{n} w_{i n} F_{i n}(x) .
$$

$F$ has moments

$$
m_{r}=m_{r}(F)=\int x^{r} d F(x)=n^{-1} \sum_{i=1}^{n} w_{i n} m_{r}\left(F_{i n}\right), \mu_{r}=\mu_{r}(F)=E_{F}\left(X-m_{1}\right)^{r}
$$

with sample versions

$$
\widehat{m}_{r}=m_{r}(\widehat{F})=n^{-1} \sum_{i=1}^{n} w_{i n} X_{i n}^{r}, \widehat{\mu}_{r}=\mu_{r}(\widehat{F})=n^{-1} \sum_{i=1}^{n} w_{i n}\left(X_{i n}-\widehat{m}_{1}\right)^{r} .
$$

For convenience we now suppress the subscript $n$ and write $X_{i}=X_{i n}, F_{i}=F_{\text {in }}, w_{i}=w_{i n}$. 
Let $T(G)$ be a smooth real functional defined for all signed measures $G(x)$ on $R^{s}$ with total measure $G(\infty)=1$. (This is the condition that requires the constraint (1.1).) The $r$ th order (von Mises) functional derivative of $T(G) T_{G}\left(x_{1}, \ldots, x_{r}\right)$ for $x_{1}, \ldots, x_{r}$ in $R^{s}$ may be defined just as in the case when $F(x)$ is a probability distribution, and the von Mises-Taylor expansion for two such signed measures $G, H$ remains valid, giving an iterative method for obtaining higher derivatives:

$$
T(G)-T(H)=\sum_{r=1}^{\infty} T_{r}(G, H) / r !,
$$

where

$$
T_{r}(G, H)=\int \ldots \int T_{H}\left(x_{1}, \ldots, x_{r}\right) d G\left(x_{1}\right) \ldots d G\left(x_{r}\right)
$$

where $T_{H}\left(x_{1}, \ldots, x_{r}\right)$ is made unique by two conditions: first that it is symmetric under permutation of arguments $x_{1}, \ldots, x_{r}$; and second that it satisfies

$$
\int T_{F}\left(x_{1}, \ldots, x_{r}\right) d F\left(x_{1}\right) \equiv 0
$$

for $r \geq 1$. The first derivative, $T_{H}(x)$ is just the coefficient of $\epsilon$ in the Taylor expansion of $T\left(H+\epsilon\left(\delta_{x}-H\right)\right)$ about $\epsilon=0$, where $\delta_{x}$ is the distribution putting weight 1 at $x$. It is sometimes called the influence function of $T(F)$. The rule for differentiating $S(H)=$ $T_{H}\left(x_{1}, \ldots, x_{r}\right)$ given by Theorem 2.1 of Withers (1983) remains valid:

$$
T_{H}\left(x_{1} \ldots x_{r+1}\right)=S_{H}\left(x_{r+1}\right)+\sum_{i=1}^{r} T_{H}\left\langle x_{1} \ldots x_{r+1}\right\rangle_{i},
$$

where $S(H)=T_{H}\left(x_{1} \ldots x_{r}\right)$ and $\langle\cdot\rangle_{i}$ means "drop the $i$ th column". For example, putting $r=1$, the second derivative is given by $T_{H}\left(x_{1} x_{2}\right)=S_{H}\left(x_{2}\right)+T_{H}\left(x_{2}\right)$, where $S(H)=T_{H}\left(x_{1}\right)$. The theory of statistical functionals was pioneered by von Mises (1947). The importance and use of the influence function has been widely used to obtain the asymptotic variance of general estimates,

$$
\operatorname{nvar}(T(\widehat{F})) \rightarrow \int T_{F}(x)^{2} d F(x)
$$

as $n \rightarrow \infty$. The second derivative has been used to estimate and correct for bias:

$$
E T(\widehat{F})-T(F)=n^{-1} \int T_{F}(x, x) d F(x) / 2+O\left(n^{-2}\right) .
$$

This was used by Jaeckel (1972) to justify the infinitesimal jackknife. However the use of other higher order derivatives has not been widespread. The reason for seems to be that not until Withers (1983), was the formula (1.5) available to compute higher order derivatives. Nevertheless their use since then has been disappointing. Perhaps this is due to a common misconception that for $T(F)$ a function of moments, it is just as easy to simply use ordinary Taylor expansions. To see that this is not true, consider the following simple example.

Example 1.1 Let us compute the asymptotic variance of the rth central sample moment, that is, $a_{21} / n$, by both the functional method and the ordinary Taylor expansion method, 
when $T(F)=\mu_{r}=\int(x-\mu)^{r} d F(x), \mu=m_{1}(F)$, observations are iid, and the ordinary unweighted empirical distribution is used. Then

$$
\begin{aligned}
& T_{x}=(x-\mu)^{r}-\mu_{r}-r(x-\mu) \mu_{r-1}, \\
& a_{21}=\int T_{x}^{2} d F(x)=\mu_{2 r}-\mu_{r}^{2}-2 \mu_{r-1} \mu_{r+1}+r^{2} \mu_{r-1}^{2} \mu_{2} .
\end{aligned}
$$

The ordinary Taylor series method writes $\mu_{r}$ as a function of the non-central moments:

$\mu_{r}=\sum_{i=0}^{r}(-1)^{r-i}\left(\begin{array}{l}r \\ i\end{array}\right) m_{i} m_{1}^{r-i}=(-1)^{r-1}(r-1) m_{1}^{r}+\sum_{i=2}^{r}(-1)^{r-i}\left(\begin{array}{l}r \\ i\end{array}\right) m_{i} m_{1}^{r-i}=t\left(m_{1}, \cdots, m_{r}\right)$ say, with derivatives $t_{\cdot i}=\partial t\left(m_{1}, \cdots, m_{r}\right) / \partial m_{i}$ given by

$$
t_{\cdot 1}=(-1)^{r-1}(r-1) r m_{1}^{r-1}+\sum_{i=2}^{r}(-1)^{r-i}\left(\begin{array}{l}
r \\
i
\end{array}\right)(r-i) m_{i} m_{1}^{r-i-1},
$$

and for $1<i \leq r$

$$
t_{\cdot i}=(-1)^{r-i}\left(\begin{array}{c}
r \\
i
\end{array}\right) m_{1}^{r-i}
$$

$\operatorname{Also} \operatorname{covar}\left(m_{i}(\widehat{F}), m_{j}(\widehat{F})\right) \approx\left(m_{i+j}-m_{i} m_{j}\right) / n$. So,

$$
a_{21}=\sum_{i, j=1}^{r} t_{i} t_{j}\left(m_{i+j}-m_{i} m_{j}\right)
$$

The challenge to these advocates of the ordinary Taylor method is to show that this reduces to (1.6). Even for the variance, this takes some time.

In Section 2 we use the von-Mises expansion for $\widehat{\theta}=T(\widehat{F})$ to obtain the basic cumulant expansion

$$
\kappa_{r}(\widehat{\theta})=\sum_{j=r-1}^{\infty} a_{r j} n^{-j}
$$

for $r \geq 1$ needed for the Edgeworth-Cornish expansions of Appendix A. (So, $a_{21} / n$ and $a_{11} / n$ are the asymptotic variance and bias of $T(\widehat{F})$, viewed as an estimator of $T(F)$.) These expansions require that the cumulant coefficients $\left\{a_{r j}\right\}$ are all bounded as $n \rightarrow \infty$. This is true if

$$
W_{r}=n^{-1} \sum_{i=1}^{n} w_{i}^{r}
$$

is bounded for $r \geq 1$ and the $[\cdot]_{i j \ldots}$ functions of Section 2 are bounded. The expansions of Appendix A also require that $a_{21}$ is bounded away from 0 . Typically this is true if $W_{2}$ is bounded away from 0 . The first order results of Koul (2002) may be reconciled with our first order results by noting that he works with $d_{i}=w_{i} /\left(n W_{2}\right)^{1 / 2}$. Throughout, we assume that all weights $\left\{w_{i n}\right\}$ are bounded and that all weight functions have finite derivatives. 


\section{Moment and Cumulant Expansions for Estimates}

What happens to non-parametric estimates when the assumption that observations come from the same distribution breaks down? Here we derive the cumulant expansion (1.7) for $\widehat{\theta}=T(\widehat{F})$, giving explicitly the cumulant coefficients of (1.7), $a_{21}, a_{11}, a_{32}, a_{22}, a_{43}$, needed for third order expansions and inference for non-iid observations.

We follow the approach of Withers (1983) deriving the cumulant expansion from the moment expansion

$$
E\{T(\widehat{F})-T(F)\}^{r}=\sum_{j \geq r / 2} a_{r j}^{\prime} n^{-j}
$$

for $r \geq 1$ using the relations between their coefficients $\left\{a_{r j}\right\}$ and $\left\{a_{r j}^{\prime}\right\}$ given in Theorem 3.1 of Withers (1983). We use the following notation with $F$ of (1.2):

$$
\begin{aligned}
T_{x_{1} \ldots x_{r}} & =T_{F}\left(x_{1}, \ldots, x_{r}\right), \\
{\left[1^{r}\right]_{i} } & =E T_{X_{i}}^{r}=\int T_{x_{1}}^{r} d F_{i}\left(x_{1}\right), \\
{\left[1^{r}, 11\right]_{i} } & =E T_{X_{i}}^{r} T_{X_{i} X_{i}}=\int T_{x_{1}}^{r} T_{x_{1} x_{1}} d F_{i}\left(x_{1}\right), \\
{\left[1^{r}, 12^{s}, 2^{t}\right]_{i j} } & =E^{i n d} T_{X_{i}}^{r} T_{X_{i} X_{j}}^{s} T_{X_{j}}^{t}=\iint T_{x_{1}}^{r} T_{x_{1} x_{2}}^{s} T_{x_{2}}^{t} d F_{i}\left(x_{1}\right) d F_{j}\left(x_{2}\right), \\
{[1,122]_{i j} } & =E^{i n d} T_{X_{i}} T_{X_{i} X_{j} X_{j}}=\iint T_{x_{1}} T_{x_{1} x_{2} x_{2}} d F_{i}\left(x_{1}\right) d F_{j}\left(x_{2}\right), \\
{[1,2,3,123]_{i j k} } & =E^{i n d} T_{X_{i}} T_{X_{j}} T_{X_{k}} T_{X_{i} X_{j} X_{k}} \\
& =\iiint T_{x_{1}} T_{x_{2}} T_{x_{3}} T_{x_{1} x_{2} x_{3}} d F_{i}\left(x_{1}\right) d F_{j}\left(x_{2}\right) d F_{k}\left(x_{3}\right)
\end{aligned}
$$

and so on, where $E^{i n d}$ means $E$ treating $X_{i}, X_{j}, \cdots$ as independent. Now set

$$
\begin{aligned}
& {\left[1^{r}\right]=n^{-1} \sum_{i=1}^{n} w_{i}^{r}\left[1^{r}\right]_{i},} \\
& {\left[1^{r}, 11\right]=n^{-1} \sum_{i=1}^{n} w_{i}^{r+2}[11]_{i},} \\
& {\left[1^{r}, 12^{s}, 2^{t}\right]=n^{-2} \sum_{i, j=1}^{n} w_{i}^{r+s} w_{j}^{s+t}\left[1^{r}, 12^{s}, 2^{t}\right]_{i j},}
\end{aligned}
$$

and more generally for $S$ a number of sequences from and including $1,2, \ldots, r$ we set

$$
[S]=n^{-r} \sum_{i_{1}=1}^{n} \ldots \sum_{i_{r}=1}^{n} w_{i_{1}}^{\lambda_{1}} \ldots w_{i_{r}}^{\lambda_{r}}[S]_{i_{1} \ldots i_{r}},
$$

where $\lambda_{j}$ is the number of times $j$ occurs in $S$.

In Appendix B we derive the following expressions for the cumulant coefficients needed 
for the Edgeworth-Cornish-Fisher expansions of $T(\widehat{F})$ to $O\left(n^{-3 / 2}\right)$ :

$$
\begin{aligned}
& a_{10}=T(F), \quad a_{21}=\left\{1^{2}\right\} \\
& a_{11}=\{11\} / 2 \text { and } a_{32}=\left\{1^{3}\right\}+3\{1,2,12\} \\
& a_{22}=\{1,11\}+\left\{12^{2}\right\} / 2+\{1,122\} \\
& a_{43}=\left\{1^{4}\right\}-3\left\{1^{2}, 1^{2}\right\}+12\left\{1,2^{2}, 12\right\}+12\{1,2,13,23\}+4\{1,2,3,123\}
\end{aligned}
$$

where

$$
\begin{aligned}
& \left\{1^{r}\right\}=n^{-1} \sum_{i=1}^{n} w_{i}^{2} \mu_{r}\left(T_{F}\left(X_{i}\right)\right) \\
& = \begin{cases}n^{-1} \sum_{i=1}^{n} w_{i}^{2}\left(\left[1^{2}\right]_{i}-[1]_{i}^{2}\right), & \text { if } r=2, \\
n^{-1} \sum_{i=1}^{n} w_{i}^{3}\left\{\left[1^{3}\right]_{i}-3[1]_{i}\left[1^{2}\right]_{i}+2[1]_{i}^{3}\right\}, & \text { if } r=3, \\
n^{-1} \sum_{i=1}^{n} w_{i}^{4}\left\{\left[1^{4}\right]_{i}-4[1]_{i}\left[1^{3}\right]_{i}\left[1^{3}\right]_{i}+6[1]_{i}^{2}\left[1^{2}\right]_{i}-3[1]_{i}^{4}\right\}, & \text { if } r=4,\end{cases} \\
& \{11\}=[11]-n^{-1} \sum_{i=1}^{n} w_{i}^{2}[12]_{i i}=n^{-1} \sum_{i=1}^{n} w_{i}^{2}\left\{[11]_{i}-[12]_{i i}\right\} \text {, } \\
& \{1,2,12\}=n^{-2} \sum_{i, j=1}^{n} w_{i}^{2} w_{j}^{2}\left([1,2,12]_{i j}-2[1]_{j}[1,12]_{i j}+[1]_{i}[1]_{j}[12]_{i j}\right) \text {, } \\
& \{1,11\}=n^{-1} \sum_{i=1}^{n} w_{i}^{3}\left([1,11]_{i}-[1]_{i}[11]_{i}-2[1,12]_{i i}+2[1]_{i}[12]_{i i}\right), \\
& \left\{12^{2}\right\}=n^{-2} \sum_{i, j=1}^{n} w_{i}^{2} w_{j}^{2}\left(\left[12^{2}\right]_{i j}-2[12,13]_{i j j}+[12]_{i j}^{2}\right), \\
& \{1,122\}=n^{-2} \sum_{i, j=1}^{n} w_{i}^{2} w_{j}^{2}\left([1,122]_{i j}-[1,123]_{i j j}-[1]_{i}[122]_{i j}+[1]_{i}[123]_{i j j}\right), \\
& \left\{1^{2}, 1^{2}\right\}=n^{-1} \sum_{i=1}^{n} w_{i}^{4} \mu_{2}\left(T_{F}\left(X_{i}\right)\right)^{2}=n^{-1} \sum_{i=1}^{n} w_{i}^{4}\left(\left[1^{2}\right]_{i}-[1]_{i}^{2}\right)^{2}, \\
& \left\{1,2^{2}, 12\right\}=n^{-2} \sum_{i, j=1}^{n} w_{i}^{2} w_{j}^{3}\left(\left[1,2^{2}, 12\right]_{i j}-\left[1^{2}\right]_{j}[1,12]_{i j}-[1]_{j}[1,2,12]_{i j}\right. \\
& \left.+2[1]_{j}^{2}[1,12]_{i j}-[1]_{i} \tau_{i j}\right), \\
& \tau_{i j}=\left[1^{2}, 12\right]_{j i}-\left[1^{2}\right]_{j}[12]_{i j}-[1]_{j}[1,12]_{j i}+2[1]_{j}^{2}[12]_{i j}, \\
& \{1,2,13,23\}=n^{-3} \sum_{i, j, k=1}^{n} w_{i}^{2} w_{j}^{2} w_{k}^{2}\left([1,2,13,23]_{i j k}-[1]_{k}[1,12,23]_{i j k}\right. \\
& \left.-[1,12]_{i j}[1,12]_{k j}+2[1]_{k}[12]_{j k}[1,12]_{i j}-[1]_{i}[1]_{k}[12]_{i j}[12]_{j k}\right), \\
& \{1,2,3,123\}=n^{-3} \sum_{i, j, k=1}^{n} w_{i}^{2} w_{j}^{2} w_{k}^{2}\left([1,2,3,123]_{i j k}-3[1]_{k}[1,2,123]_{i j k}\right. \\
& \left.+3[1]_{j}[1]_{k}[1,123]_{i j k}-[1]_{i}[1]_{j}[1]_{k}[123]_{i j k}\right) .
\end{aligned}
$$

In addition the following coefficient is useful for the calculation of the second order bias:

$$
a_{12}=\{111\} / 6+\{1122\} / 8,
$$


where

$$
\{111\}=n^{-1} \sum_{i=1}^{n} w_{i}^{3}\left([111]_{i}-3[122]_{i i}+2[123]_{i i i}\right)
$$

and

$$
\{1122\}=n^{-2} \sum_{i, j=1}^{n} w_{i}^{2} w_{j}^{2}\left([1122]_{i j}-2[1233]_{i i j}+[1234]_{i i j j}\right) .
$$

Typically $a_{21}$ is bounded away from 0 if and only if $W_{2}$ is bounded away from 0 .

For iid observations, (2.1) $-(2.5)$ reduce to

$$
\begin{aligned}
a_{10}= & T(F), \quad a_{21}=\left[1^{2}\right]=W_{2}\left[1^{2}\right]_{1} \\
a_{11}= & {[11] / 2=W_{2}[11]_{1} / 2 } \\
a_{32}= & {\left[1^{3}\right]+3[1,2,12]=W_{3}\left[1^{3}\right]_{1}+3 W_{2}^{2}[1,2,12]_{11}, } \\
a_{22}= & {[1,11]+\left[12^{2}\right] / 2+[1,122] } \\
= & W_{3}[1,11]_{1}+W_{2}^{2}\left[12^{2}\right]_{11} / 2+W_{2}^{2}[1,122]_{11}, \\
a_{43}= & {\left[1^{4}\right]-3 W_{4}\left[1^{2}\right]_{1}^{2}+12\left[1,12,2^{2}\right]+12[1,2,23,31]+4[1,2,3,123] } \\
= & W_{4}\left\{\left[1^{4}\right]_{1}-3\left[1^{2}\right]_{1}^{2}\right\}+12 W_{2} W_{3}\left[1,12,2^{2}\right]_{11} \\
& +4 W_{2}^{3}\left\{3[1,2,23,31]_{111}+[1,2,3,123]_{111}\right\} \\
a_{12}= & {[111] / 6+[1122] / 8=W_{3}[111]_{1} / 6+W_{2}^{2}[1122]_{11} / 8, }
\end{aligned}
$$

where $W_{r}$ is given by (1.8). This follows from the above results and (1.4). For $w_{\text {in }} \equiv 1$ these reduce to the expressions of Theorem 3.1 of Withers (1983).

\section{Two Simple Examples}

If $T(F)$ is a polynomial in $F$ of degree $r$, (for example, $\mu_{r}(F)$ ), then derivatives of order greater than $r$ are zero. Let us work through two simple examples: the mean and the variance.

Example 3.1 Suppose that $T(F)=\mu(F)=\mu$ say, and $s=1$. Then $T_{x}=x-\mu$, and higher derivatives are zero. Then

$$
a_{r, r-1}=n^{-1} \sum_{i=1}^{n} w_{i}^{r} \kappa_{r}\left(X_{i}\right)
$$

and other cumulant coefficients are 0 . If the observations are iid, then $a_{r, r-1}=W_{r} \kappa_{r}(X)$.

Now suppose that $\left\{w_{i}, F_{i}(x)\right\}$ can be parameterised as $w_{i n}=w(i / n), F_{i n}(x)=F(x, i / n)$ for some smooth functions $w(t), F(x, t)$. That is, $X_{i n}$ has distribution $G_{\theta(i / n)}(x)$ say, and $F(x, t)=G_{\theta(t)}(x)$. Then we can write

$$
\begin{aligned}
& E X_{i n}^{r}=m_{r}(i / n), \text { where } m_{r}(t)=\int x^{r} F(d x, t), \\
& \kappa_{r}\left(X_{\text {in }}\right)=\kappa_{r}(i / n), \text { where } \kappa_{1}(t)=m_{1}(t), \kappa_{2}(t)=m_{2}(t)-m(t)^{2}, \cdots \\
& a_{r, r-1}=n^{-1} \sum_{i=1}^{n} k_{r}(i / n), \text { where } k_{r}(t)=w(t)^{r} \kappa_{r}(t) .
\end{aligned}
$$


That is, $m_{r}(t)$ and $\kappa_{r}(t)$ are the rth moment and cumulant of $G_{\theta(t)}(x)$. By the EulerMcLaurin expansion (Abramowitz and Stegun, 1964, Equation (23.1.30), page 806),

$$
\begin{aligned}
& n^{-1} \sum_{i=1}^{n} g(i / n)=\sum_{k=0}^{\infty} \alpha_{k}(g) n^{-k}, \text { where } \\
& \alpha_{0}(g)=\int_{0}^{1} g(t) d t \\
& \alpha_{1}(g)=\{g(1)-g(0)\} / 2, \\
& \alpha_{k}(g)=\left\{g^{(k-1)}(1)-g^{(k-1)}(0)\right\} B_{k} / k ! \text { for } k=2,3, \ldots
\end{aligned}
$$

and $B_{k}$ is the kth Bernoulli number, given by Abramowitz and Stegun (1964, page 809, last column): $B_{1}=-1 / 2, B_{2}=1 / 6, B_{3}=0, B_{4}=-1 / 30, \cdots$ and $B_{k}=0$ for $k=3,5,7, \cdots$. Applying this to $g=k_{r}$ of (3.1), we see that the rth cumulant of $\mu(\widehat{F})$ satisfies the basic expansion (1.7) with the new coefficients $a_{r j}^{\prime}=\alpha_{j+1-r}\left(k_{r}\right)$. So, the leading coefficients are

$$
a_{r, r-1}^{\prime}=\int_{0}^{1} k_{r}(t) d t, a_{r r}^{\prime}=\left(k_{r}(1)-k_{r}(0)\right) / 2
$$

In particular

$$
a_{10}^{\prime}=\int_{0}^{1} w(t) d t \int x F(d x, t), a_{21}^{\prime}=\int_{0}^{1} w(t)^{2} \kappa_{2}(t) d t .
$$

So, for the unweighted case $w(t)=1$,

$$
a_{10}^{\prime}=\int_{0}^{1} d t \int x F(d x, t), a_{21}^{\prime}=\int_{0}^{1} \kappa_{2}(t) d t
$$

Example 2.1.1 Suppose that $G_{\theta}(x)=1-e^{-x / \theta}$ on $(0, \infty)$, the scaled exponential distribution. Then

$$
\begin{aligned}
& \kappa_{r}(t)=(r-1) ! \theta(t)^{r}, k_{r}(t)=(r-1) ! \eta(t)^{r}, \text { where } \eta(t)=w(t) \theta(t), \\
& a_{r, r-1}^{\prime}=(r-1) ! \int_{0}^{1} \eta(t)^{r} d t, a_{r r}^{\prime}=(r-1) !\left[\eta(1)^{r}-\eta(0)^{r}\right] / 2
\end{aligned}
$$

So, to this degree of approximation, weighting the observations amounts to weighting the scale parameter, $\theta(t)$.

Since $\mu(F)$ is linear in $F$, the last example did not need the machinery of functional differentiation. But as pointed out in Example 1.1, this is not the case for the next example, the variance.

Example 3.2 Suppose that $s=1$ and $T(F)=\mu_{2}(F)=\mu_{2}$ say. Then $T_{x}=\mu_{x}^{2}-\mu_{2}$, where $\mu_{x}=x-\mu, T_{x y}=-2 \mu_{x} \mu_{y}$, and higher derivatives are zero. We give the cumulant coefficients in terms of

$$
M_{r s \cdots}^{k}=n^{-1} \sum_{i=1}^{n} w_{i}^{k} \mu_{r i} \mu_{s i} \cdots
$$


where $\mu_{r i}=E \mu_{X_{i}}^{r}=E\left(X_{i}-\mu\right)^{r}$. Since

$$
\left\{1^{r}\right\}=n^{-1} \sum_{i=1}^{n} w_{i}^{r} \mu_{r}\left(\mu_{X_{i}}^{2}\right),
$$

we have $a_{21}=\left\{1^{2}\right\}=M_{4}^{2}-M_{22}^{2},\left\{1^{3}\right\}=M_{6}^{3}-3 M_{24}^{3}+2 M_{222}^{3}$ and $\left\{1^{4}\right\}=M_{8}^{4}-4 M_{26}^{4}+$ $6 M_{224}^{4}-3 M_{2222}^{4}$. Also $[11]_{i}=-2 E \mu_{X_{i}}^{2}=-2 \mu_{2 i},[12]_{i j}=-2 \mu_{1 i} \mu_{1 j}$, so that

$$
a_{11}=-M_{2}^{2}+M_{11}^{2} \text {. }
$$

Also $[1]_{i}=\mu_{2 i}-\mu_{2},[1,12]_{i j}=-2\left(\mu_{3 i}-\mu_{2} \mu_{1 i}\right) \mu_{1 j},[1,2,12]_{i j}=-2\left(\mu_{3 i}-\mu_{2} \mu_{1 i}\right)\left(\mu_{3 j}-\mu_{2} \mu_{1 j}\right)$, giving $\{1,2,12\}=-2\left(M_{3}^{2}-M_{12}^{2}\right)^{2}$, so that

$$
a_{32}=M_{6}^{3}-3 M_{24}^{3}+2 M_{222}^{3}-6\left(M_{3}^{2}-M_{12}^{2}\right)^{2} .
$$

Also $\{1,11\}=-2\left(M_{4}^{3}-2 M_{13}^{3}-M_{22}^{3}+2 M_{112}^{3}\right),\left\{12^{2}\right\}=4\left(M_{2}^{2}-M_{11}^{2}\right)^{2},\{1,122\}=0$, so that

$$
a_{22} / 2=\left(M_{2}^{2}-M_{11}^{2}\right)^{2}-M_{4}^{3}+M_{22}^{3}+2 M_{13}^{3}-2 M_{112}^{3} .
$$

Similarly writing (2.4) as $a_{43}=b_{1}-3 b_{2}+12 b_{3}+12 b_{4}+4 b_{5}$, one obtains

$$
\begin{aligned}
b_{1}= & M_{8}^{4}-4 M_{26}^{4}+6 M_{224}^{4}-3 M_{2222}^{4}, \\
b_{2}= & M_{44}^{4}-2 M_{224}^{4}+M_{2222}^{4}, \\
b_{3}= & 2 M_{3}^{3}\left(-M_{5}^{3}+M_{14}^{3}+M_{23}^{3}-4 M_{122}^{3}+\mu_{2} M_{3}^{3}+5 \mu_{2}^{2} M_{1}^{3}\right) \\
& +2 M_{12}^{2}\left[2 M_{5}^{3}-M_{23}^{3}+2 M_{122}^{3}-3 \mu_{2}\left(M_{3}^{3}+M_{12}^{3}\right)+3 \mu_{2}^{2} M_{1}^{3}\right] \\
& -2 M_{1}^{2} \mu_{2}\left[M_{5}^{3}+M_{14}^{3}-2 M_{122}^{3}-2 \mu_{2}\left(M_{3}^{3}+M_{12}^{3}\right)+8 \mu_{2}^{2} M_{1}^{3}\right], \\
b_{4}= & 4\left(M_{3}^{2}-\mu_{2} M_{1}^{2}\right)\left(M_{3}^{2}-M_{12}^{2}\right)\left(M_{2}^{2}-M_{11}^{2}\right), \\
b_{5}= & 0 .
\end{aligned}
$$

Specialising to the iid case gives $\mu_{r i}=\mu_{r}$,

$$
\begin{aligned}
& {\left[1^{2}\right]_{1}=\mu_{4}-\mu_{2}^{2},[11]_{1}=-2 \mu_{2},\left[1^{3}\right]_{1}=\mu_{6}-3 \mu_{2} \mu_{4}+2 \mu_{2}^{3},} \\
& {[1,2,12]_{11}=-2 \mu_{3}^{2},[1,11]_{1}=-2\left(\mu_{4}-\mu_{2}^{2}\right)} \\
& {\left[12^{2}\right]_{11}=4 \mu_{2}^{2},\left[1^{4}\right]_{1}=\mu_{8}-4 \mu_{2} \mu_{6}+6 \mu_{2}^{2} \mu_{4}-3 \mu_{2}^{4}} \\
& {\left[1,12,2^{2}\right]_{11}=-2 \mu_{3}\left(\mu_{5}-2 \mu_{2} \mu_{3}\right),[1,2,23,31]_{111}=4 \mu_{2} \mu_{3}^{2}} \\
& {[1,2,3,123]_{111}=[1,122]_{11}=[111]_{1}=[1122]_{11}=0}
\end{aligned}
$$

so that

$$
\begin{aligned}
a_{21}= & W_{2}\left(\mu_{4}-\mu_{2}^{2}\right), a_{11}=-W_{2} \mu_{2}, \\
a_{32}= & W_{3}\left(\mu_{6}-3 \mu_{2} \mu_{4}+2 \mu_{2}^{3}\right)-6 W_{2}^{2} \mu_{3}^{2}, \\
a_{22}= & -2 W_{3}\left(\mu_{4}-\mu_{2}^{2}\right)+2 W_{2}^{2} \mu_{2}^{2}, \\
a_{43}= & W_{4}\left[\mu_{8}-4 \mu_{2} \mu_{6}+6 \mu_{2}^{2} \mu_{4}-3 \mu_{2}^{4}-3\left(\mu_{4}-\mu_{2}^{2}\right)^{2}\right]-24 W_{2} W_{3} \mu_{3}\left(\mu_{5}-2 \mu_{2} \mu_{3}\right) \\
& +48 W_{2}^{3} \mu_{2} \mu_{3}^{2} .
\end{aligned}
$$

For the unweighted case, these cumulant coefficients $\left\{a_{r i}\right\}$, reduce to those of Example 2 of Withers (1983). 


\section{A Chain Rule for More Complex Examples}

When $T(F)$ is a function of moments (for example, the coefficient of variation or correlation), the following chain rule is very useful.

Suppose that $f: R^{p} \rightarrow R$ is a smooth function and that $S(F)$ is a smooth functional in $R^{p}$. For $1 \leq a \leq p$, let $S_{a \cdot x_{1} \cdots x_{r}}$ denote the $r$ th derivatives of $S_{a}(F)$. Then by an extension of the chain rule for differentiating a function of a function, the first three derivatives of $T(F)=f(S(F))$ are

$$
\begin{aligned}
& T_{x}=f_{a} S_{a x}, T_{x_{1} x_{2}}=f_{a} S_{a \cdot x_{1} x_{2}}+f_{a b} S_{a \cdot x_{1}} S_{b \cdot x_{2}}, \\
& T_{x_{1} x_{2} x_{3}}=f_{a} S_{a \cdot x_{1} x_{2} x_{3}}+f_{a b} \sum_{123}^{3} S_{a \cdot x_{1}} S_{b \cdot x_{2} x_{3}}+f_{a b c} S_{a \cdot x_{1}} S_{b \cdot x_{2}} S_{c \cdot x_{3}},
\end{aligned}
$$

where $f_{a}=f_{a_{1} \cdots a_{r}}=\partial^{r} f(s) /\left.\partial s_{1} \cdots \partial s_{r}\right|_{s=S(F)}$, we use the tensor summation convention of implicit summation of pairs of $a, b, \cdots$, and $\sum_{123}^{3}$ sums over all 3 permutations of 1,2,3 giving distinct terms. Set

$$
\nu_{i}^{a b \cdots}=E S_{a X_{i}} S_{b X_{i}} \cdots \text { and } M^{k}(a b \cdots, c d \cdots, \cdots)=n^{-1} \sum_{i=1}^{n} w_{i}^{k} \nu_{i}^{a b \cdots} \nu_{i}^{c d \cdots} \cdots .
$$

Then

$$
\begin{aligned}
& {[1]_{i}=f_{a} \nu_{i}^{a}, \quad\left[1^{2}\right]_{i}=f_{a} f_{b} \nu_{i}^{a b},} \\
& {\left[1^{3}\right]_{i}=f_{a} f_{b} f_{c} \nu_{i}^{a b c},} \\
& {[11]_{i}=f_{a b} \nu_{i}^{a b}+f_{a} E S_{a X_{i} X_{i}},} \\
& {[12]_{i j}=f_{a b} \nu_{i}^{a} \nu_{j}^{b}+f_{a} E^{i n d} S_{a X_{i} X_{j}},} \\
& {[1,12]_{i j}=f_{c}\left(f_{a b} \nu_{i}^{a c} \nu_{j}^{b}+f_{a} E^{i n d} S_{c X_{i}} S_{a X_{i} X_{j}}\right),} \\
& {[1,2,12]_{i j}=f_{a} f_{b}\left(f_{c d} \nu_{i}^{a c} \nu_{j}^{b d}+f_{c} E^{i n d} S_{a X_{i}} S_{b X_{j}} S_{c X_{i} X_{j}}\right) .}
\end{aligned}
$$

Set

$$
\begin{aligned}
& C(a b)=\mu\left(S_{a X_{i}}, S_{b X_{i}}\right)=M^{2}(a b)-M^{2}(a, b), \\
& C(a b c)=n^{-1} \sum_{i=1}^{n} w_{i}^{3} \mu\left(S_{a X_{i}}, S_{b X_{i}}, S_{c X_{i}}\right)=M^{3}(a b c)-\sum_{a b c}^{3} M^{3}(a b, c)+2 M^{3}(a, b, c)
\end{aligned}
$$

since

$$
\mu\left(S_{a X_{i}}, S_{b X_{i}}, S_{c X_{i}}\right)=\nu_{i}^{a b c}-\sum_{a b c}^{3} \nu_{i}^{a b} \nu_{i}^{c}+2 \nu_{i}^{a} \nu_{i}^{b} \nu_{i}^{c}
$$

Then

$$
\begin{aligned}
& a_{21}=f_{a} f_{b} C(a b), \\
& 2 a_{11}=f_{a b} C(a b)+f_{a} n^{-1} \sum_{i=1}^{n} w_{i}^{2}\left(E S_{a X_{i} X_{i}}-\left.E^{i n d} S_{a X_{i} X_{j}}\right|_{j=i}\right) .
\end{aligned}
$$


Also $a_{32}$ is given by (2.2) in terms of

$$
\begin{aligned}
\left\{1^{3}\right\}= & f_{a} f_{b} f_{c} C(a b c), \\
\{1,2,12\}= & f_{a} f_{b} f_{c} n^{-2} \sum_{i, j=1}^{n} w_{i}^{2} w_{j}^{2} E^{i n d} S_{a X_{i} X_{j}}\left(S_{b X_{i}} S_{c X_{j}}-2 \nu_{j}^{b} S_{c X_{i}}+\nu_{i}^{b} \nu_{j}^{c}\right) \\
& +f_{a} f_{b} f_{c d} C(a d) C(b c) .
\end{aligned}
$$

For the iid case these reduce to

$$
\begin{aligned}
& a_{21}=W_{2} f_{a} f_{b} \nu_{1}^{a b}, \\
& a_{11}=W_{2}\left(f_{a b} \nu_{1}^{a b}+f_{a} E S_{a X X}\right) / 2, \\
& a_{32}=f_{a} f_{b} f_{c}\left(W_{3} \nu_{1}^{a b c}+3 W_{2}^{2} E^{i n d} S_{a X_{1} X_{2}} S_{b X_{1}} S_{c X_{2}}\right)+3 f_{a} f_{b} f_{c d} W_{2}^{2} \nu_{1}^{a d} \nu_{1}^{b c} .
\end{aligned}
$$

Example 4.1 Suppose that $s=1, p=2, S_{1}(F)=\mu, S_{2}(F)=\mu_{2}$. After some simplification one obtains

$$
\begin{aligned}
& a_{21}=f_{a} f_{b} C(a b)=f_{1}^{2} C(11)+2 f_{1} f_{2} C(12)+f_{2}^{2} C(22) \\
& 2 a_{11}=f_{a b} C(a b)-2 f_{2} C(11)=\left(f_{11}-2 f_{2}\right) C(11)+2 f_{12} C(12)+f_{22} C(22), \\
& a_{32}=\left\{1^{3}\right\}+3\{1,2,12\}
\end{aligned}
$$

where

$$
\begin{aligned}
& \left\{1^{3}\right\}=f_{1}^{3} C(111)+3 f_{1}^{2} f_{2} C(112)+3 f_{1} f_{2}^{2} C(122)+f_{2}^{3} C(222), \\
& \{1,2,12\}=D_{1}^{2}\left(f_{11}-f_{2}\right)+2 D_{1} D_{2} f_{12}+D_{2}^{2} f_{12}, D_{j}=f_{a} C(j a) .
\end{aligned}
$$

Also, in the notation of (3.2), $C(a b), C(a b c)$ reduce to

$$
\begin{aligned}
& C(a b)=M_{a+b}^{2}-M_{a b}^{2} ; \\
& C(a b c)=M_{a+b+c}^{2}-\sum_{a b c}^{3} M_{a+b, c}^{2}+2 M_{a, b, c}^{2}: \\
& C(111)=M_{3}^{3}, C(112)=M_{4}^{3}-M_{22}^{3}-2 M_{13}^{3}+2 M_{112}^{3}, \\
& C(122)=M_{5}^{3}-M_{14}^{3}-2 M_{23}^{3}+2 M_{122}^{3}, C(222)=M_{6}^{3}-3 M_{24}^{3}+2 M_{222}^{3} .
\end{aligned}
$$

For the iid case,

$$
\begin{aligned}
& C(11)=W_{2} \mu_{2}, C(12)=W_{2} \mu_{3}, C(22)=W_{2}\left(\mu_{4}-\mu_{2}^{2}\right), \\
& C(111)=W_{3} \mu_{3}, C(112)=W_{3}\left(\mu_{4}-\mu_{2}^{2}\right), \\
& C(122)=W_{3}\left(\mu_{5}-2 \mu_{2} \mu_{3}\right), C(222)=W_{3}\left(\mu_{6}-3 \mu_{2} \mu_{4}+2 \mu_{2}^{3}\right) .
\end{aligned}
$$

We now give two applications of this example, the Studentised mean and the coefficient of variation. Set $\sigma=\sigma(F)=\mu_{2}^{1 / 2}$ and $\lambda_{r}=\mu_{r} / \mu_{2}^{r / 2}$.

Example 4.2 The Studentized mean. Take $s=1, T(G)=[\mu(G)-\mu(F)] / \sigma(G)$. Then in terms of $C(a b), C(a b c)$ of Example 2.4,

$$
\begin{aligned}
& T(F)=f_{2}=f_{11}=f_{22}=0, f_{1}=\sigma^{-1}, f_{12}=-\sigma^{-3} / 2, \\
& a_{21}=C(11) / \mu_{2}, a_{11}=-\sigma^{-3} C(12) / 2, \\
& a_{32}=C(111) \sigma^{-3}-3 C(11) C(12) \sigma^{-5} .
\end{aligned}
$$


For the iid case, $a_{21}=W_{2}, a_{11}=-W_{2} \lambda_{3} / 2, a_{32}=\left(W_{3}-3 W_{2}\right)^{2} \lambda_{3}$. For the unweighted case, this gives $a_{21}=1, a_{11}=-\lambda_{3} / 2, a_{32}=-2 \lambda_{3}$, in agreement with Withers (1989, Example 1.2, page 300).

Example 4.3 The inverse of the coefficient of variation. Suppose that $s=1$ and $T(F)=\mu / \sigma$. Using the notation of the previous two examples, we obtain

$$
\begin{aligned}
& a_{21}=C(11) / \mu_{2}-C(12) \mu / \mu_{2}^{2}+C(22) \mu^{2} / 4 \mu_{2}^{3}, \\
& 2 a_{11}=(C(11) \mu-C(12)) \sigma^{-3}+3 C(22) \mu \sigma^{-5} / 4, \\
& a_{32}=\left\{1^{3}\right\}+3\{1,2,12\},
\end{aligned}
$$

where

$$
\begin{aligned}
& \left\{1^{3}\right\}=C(111) \sigma^{-3}-3 C(112) \mu \sigma^{-5} / 2+3 C(122) \mu^{2} \sigma^{-7} / 4-C(222) \mu^{3} \sigma^{-9} / 8, \\
& \{1,2,12\}=\left(D_{1}^{2} \mu-D_{1} D_{2}\right) \sigma^{-3}+3 D_{2}^{2} \mu \sigma^{-5} / 4, \\
& D_{1}=\left[C(11)-C(12) \mu / 2 \mu_{2}\right] \sigma^{-1}, D_{2}=\left[C(12)-C(22) \mu / 2 \mu_{2}\right] \sigma^{-1} .
\end{aligned}
$$

For the iid case,

$$
\begin{aligned}
& D_{1}=W_{2} \sigma \alpha_{1}, \text { where } \alpha_{1}=1-\lambda_{3} T(F) / 2, \\
& D_{2}=W_{2} \sigma^{2} \alpha_{2}, \text { where } \alpha_{2}=\lambda_{3}-\left(\lambda_{4}-1\right) T(F) / 2,
\end{aligned}
$$

and

$$
\begin{aligned}
& a_{21}=W_{2}\left[1-\lambda_{3} T(F)+\left(\lambda_{4}-1\right) T(F)^{2} / 4\right], \\
& 2 a_{11}=W_{2}\left[T(F)\left(1+3 \lambda_{4}\right) / 4-\lambda_{3}\right], \\
& \left\{1^{3}\right\} / W_{3}=\lambda_{3}-3 T(F)\left(\lambda_{4}-1\right) / 2+3 T(F)^{2}\left(\lambda_{5}-2 \lambda_{3}\right) / 4-T(F)^{3}\left(\lambda_{6}-3 \lambda_{4}+2\right) / 8, \\
& \{1,2,12\} / W_{2}^{2}=\alpha_{1}^{2} T(F)-\alpha_{1} \alpha_{2}+3 \alpha_{2}^{2} T(F), \\
& a_{32}=\left\{1^{3}\right\}+3\{1,2,12\} .
\end{aligned}
$$

\section{References}

[1] Abramowitz, M. and Stegun, I. A. (1964). Handbook of Mathematical Functions. National Bureau of Standards, Washington D.C.

[2] Chernoff, H. and Zacks, S. (1964). Estimating the current mean of a normal distribution which is subjected to changes in time. Annals of Mathematical Statistics, 35, 999-1018.

[3] Hajek, J. and Sidak, Z. (1967) Theory of Rank Tests, Academic Press, Inc., New York.

[4] Jaeckel, L. A. (1972). The infinitesmal jackknife. Bell Laboratories Technical Report MM 72-1215-11, June 30, 1972, New Jersey.

[5] Kander, Z. and Zacks, S. (1966). Test procedures for possible changes in parameters of statistical distributions occurring at unknown time points. Annals of Mathematical Statistics, 37, 1196-1210. 
[6] Koul, H. L. (1992). Weighted empiricals and linear models. Institute of Mathematical Statistics Monograph Series, 21, Michigan State University.

[7] Koul, H. L. (2002). Weighted empirical processes in dynamic nonlinear models. Second edition. Lecture Notes in Statistics, 166, Springer, New York.

[8] Lahiri, S. N. (1992a). On bootstrapping M-estimators. Sankhyā, A, 54, 157-170.

[9] Lahiri, S. N. (1992b). Edgeworth expansions for M-estimators of a linear regression parameter. Journal of Multivariate Analysis, 43, 125-132.

[10] Lahiri, S. N. (1992c). Bootstrapping M-estimators of a multiple linear regression parameter. Annals of Statistics, 20, 1548-1570.

[11] Lahiri, S. N. (2003). Resampling Methods for Dependent Data. Springer, New York.

[12] Withers, C. S. (1983). Expansions for the distribution and quantiles of a regular functional of the empirical distribution with applications to nonparametric condidence intervals. Annals of Statistics, 11, 577-587.

[13] Withers, C. S. (1988). Nonparametric confidence intervals for functions of several distributions. Annals of the Institute of Statistical Mathematics, 40, 727-746.

[14] Withers, C. S. (1989). The distribution and cumulants of a Studentised statistic. Communications in Statistics - Theory and Methods, 18, 295-318.

[15] Withers, C. S. and Nadarajah, S. (2008). Edgeworth expansions for functions of weighted empirical distributions with applications to nonparametric confidence intervals. Journal of Nonparametric Statistics, 20, 751-768.

[16] von Mises, R. (1947). On the asymptotic distribution of differentiable statistical functionals. Annals of Mathematical Statistics, 18, 309-348. 


\section{Appendix A: Edgeworth-Cornish-Fisher Expansions}

Since the cumulants of $T(\widehat{F})$ satisfy the expansion (1.7), it follows by Withers (1984) that its standardized version, $Y_{n}=n^{1 / 2} a_{21}^{-1 / 2}\{T(\widehat{F})-T(F)\}$, has the Edgeworth-Cornish-Fisher expansions

$$
\begin{aligned}
& P_{n}(x)=P\left(Y_{n} \leq x\right) \approx \Phi(x)-\phi(x) \sum_{r=1}^{\infty} h_{r}(x) n^{-r / 2} \\
& P\left(\left|Y_{n}\right| \leq x\right) \approx 2 \Phi(x)-1-2 \phi(x) \sum_{r=1}^{\infty} h_{2 r}(x) n^{-r} \\
& \Phi^{-1}\left(P_{n}(x)\right) \approx x-\sum_{r=1}^{\infty} f_{r}(x) n^{-r / 2} \\
& P_{n}^{-1}(\Phi(x)) \approx x+\sum_{r=1}^{\infty} g_{r}(x) n^{-r / 2}
\end{aligned}
$$

where $\Phi$ and $\phi$ are the distribution and density of a unit normal random variable and $\left\{h_{r}, f_{r}, g_{r}\right\}$ are certain polynomials given by (4.1) of Withers (1984) in terms of the standardized cumulant coefficients $A_{r i}=a_{21}^{-r / 2} a_{r i}$. In terms of the Hermite polynomial $H e_{i}(x)=$ $\phi(x)^{-1}(-d / d x)^{i} \phi(x)$, the first few are given by

$$
\begin{aligned}
h_{1}= & f_{1}=g_{1}=A_{11}+A_{32} H e_{2} / 6, \\
h_{2}= & \left(A_{11}^{2}+A_{22}\right) H e_{1} / 2+\left(4 A_{11} A_{32}+A_{43}\right) H e_{3} / 24+A_{32}^{2} H e_{5} / 72, \\
h_{3}= & A_{12}+\left(A_{11}^{3}+3 A_{11} A_{22}+A_{33}\right) H e_{2} / 6 \\
& +\left(10 A_{11}^{2} A_{32}+5 A_{11} A_{43}+10 A_{22} A_{32}+A_{54}\right) H e_{1} / 120 \\
& +\left(2 A_{11} A_{32}^{2}+A_{32} A_{43}\right) H e_{6} / 144+A_{32} H e_{8} / 1296, \\
f_{2}(x)= & \left(\ell_{2} / 2-\ell_{1} \ell_{3} / 3\right) x+\ell_{4}\left(x^{3}-3 x\right) / 24-\ell_{3}^{2}\left(4 x^{3}-7 x\right) / 36, \\
f_{3}(x)= & -\ell_{2} \ell_{2} / 2+\ell_{1}^{2} \ell_{3} / 6-\ell_{2} \ell_{3}\left(5 x^{2}-3\right) / 12-\ell_{1} \ell_{4}\left(x^{2}-1\right) / 8 \\
& +\ell_{5}\left(x^{4}-6 x^{2}+3\right) / 120+\ell_{1} \ell_{3}^{2}\left(12 x^{2}-7\right) / 36-\ell_{3} \ell_{4}\left(11 x^{4}-42 x^{2}+15\right) / 144 \\
& +\ell_{3}^{3}\left(69 x^{4}-187 x^{2}+52\right) / 648+A_{12}+A_{33}\left(x^{2}-1\right) / 6, \\
g_{2}(x)= & \ell_{2} x / 2+\ell_{4}\left(x^{3}-3 x\right) / 24-\ell_{3}^{2}\left(2 x^{3}-5 x\right) / 36, \\
g_{3}(x)= & -\ell_{2} \ell_{3}\left(x^{2}-1\right) / 6+\ell_{5}\left(x^{4}-6 x^{2}+3\right) / 120-\ell_{3} \ell_{4}\left(x^{4}-5 x^{2}+2\right) / 24 \\
& +\ell_{3}^{3}\left(12 x^{4}-53 x^{2}+17\right) / 324+A_{12}+A_{33}\left(x^{2}-1\right) / 6,
\end{aligned}
$$

where $\ell_{1}=A_{11}, \ell_{2}=A_{22}, \ell_{3}=A_{32}, \ell_{4}=A_{43}$ and $\ell_{5}=A_{54}$. The coefficients given in $\S 2$ give $\left\{h_{r}, f_{r}, g_{r}, 1 \leq r \leq 2\right\}$ and so give the distribution and quantiles of $Y_{n}$ to $O\left(n^{-3 / 2}\right)$. The same is true of its density, since we can write

$$
h_{r}(x)=\sum_{i=1}\left\{h_{r i} H e_{i}(x): r+i \text { odd }\right\}
$$

so

$$
P_{n}(x) \approx \Phi(x)-\sum_{r=1}^{\infty} n^{-r / 2} \sum_{i=1}^{3 r-1} h_{r i}(-\partial / \partial x)^{i} \phi(x)
$$


giving density

$$
p_{n}(x) \approx \phi(x)\left\{1+\sum_{r=1}^{\infty} n^{-r / 2} \bar{h}_{r}(x)\right\}
$$

where

$$
\bar{h}_{r}(x)=\sum_{i=1}^{3 r-1}\left\{h_{r i} H e_{i+1}(x): r+i \text { odd }\right\}
$$

So, $\bar{h}_{1}=A_{11} H e_{1}+A_{32} H e_{3} / 6, \bar{h}_{2}=\left(A_{11}^{2}+A_{22}\right) H e_{2} / 2+\left(4 A_{11} A_{32}+A_{43}\right) H e_{4} / 24+$ $A_{32}^{2} \mathrm{He}_{6} / 72$, and so on.

Suppose $T(F)$ is $q$-variate and the weights $p$-variate. Then the distribution and density of $Y_{n}=n^{1 / 2}\{T(\widehat{F})-T(F)\}$ have the multivariate Edgeworth expansions for $x$ in $R^{q}$

$$
P_{n}(x)=P\left(Y_{n} \leq x\right) \approx \sum_{r=0}^{\infty} n^{-r / 2} \widetilde{P}_{r}(-\partial / \partial x) \Phi_{V}(x)
$$

and

$$
p_{n}(x)=\left(\partial / \partial x_{1}\right) \ldots\left(\partial / \partial x_{q}\right) P\left(Y_{n} \leq x\right) \approx \sum_{r=0}^{\infty} n^{-r / 2} \widetilde{P}_{r}(-\partial / \partial x) \phi_{V}(x)
$$

where $\Phi_{V}(x)$ and $\phi_{V}(x)$ are the distribution and density of $\mathcal{N}(0, V), V=\left(a_{1}^{\alpha \beta}\right)$,

$$
\begin{aligned}
& \widetilde{P}_{0}(t)=1, \\
& \widetilde{P}_{1}(t)=a_{1}^{\alpha} t_{\alpha}+a_{1}^{\alpha \beta \gamma} t_{\alpha} t_{\beta} t_{\gamma} / 6, \\
& \widetilde{P}_{2}(t)=a_{2}^{\alpha \beta} t_{\alpha} t_{\beta} / 2+a_{3}^{\alpha \beta \gamma \delta} t_{\alpha} t_{\beta} t_{\gamma} t_{\delta} / 24+\widetilde{P}_{1}(t)^{2},
\end{aligned}
$$

and so on, again using implicit summation over repeated pairs of indices. 


\section{Appendix B: Proofs for Section 2}

Here we derive the results of Section 2. By the von Mises expansion (1.3) and (1.4),

$$
T(\widehat{F})-T(F)=\sum_{r=1}^{\infty} T_{(r)} / r !
$$

where $T_{(r)}=T_{r}(\widehat{F}, F)=O_{p}\left(n^{-r / 2}\right)$ for $T(F)$ regular. We may express the moments of $T(\widehat{F})-T(F)$ in terms of $\alpha_{r s \ldots}=E T_{(r)} T_{(s)} \ldots=O\left(n^{-\nu}\right)$, where $\nu=R / 2$ for $R$ even, $\nu=(R+1) / 2$ for $R$ odd and $R=r+s+\ldots$ Since

$$
T_{(r)}=n^{-r} \sum_{i_{1}, \ldots, i_{r}=1}^{n} w_{i_{1}} \ldots w_{i_{r}} \int \ldots \int T_{x_{1} \ldots x_{r}} d I_{i_{1} 1} \ldots d I_{i_{r} r}
$$

where $I_{i r}=I\left(X_{i} \leq x_{r}\right)-F_{i}\left(x_{r}\right)$, to simplify $\alpha_{r s \ldots}$ we need expressions for $D_{i 1 \ldots r}=$ $E I_{i 1} \ldots I_{i r}$. Set

$$
\begin{aligned}
& \delta_{i j} \ldots=I(i=j=\ldots), \\
& \delta_{i j \ldots, k \ell \ldots}=I(i=j=\ldots \neq k=\ell=\ldots), \\
& \delta_{i j, k \ell, m n}=I(i=j, k=\ell, m=n \text { all three distinct }),
\end{aligned}
$$

and so on. Then

$$
\begin{aligned}
& E I_{i 1} I_{j 2}=\delta_{i j} D_{i 12}, \\
& E I_{i 1} I_{j 2} I_{k 3}=\delta_{i j k} D_{i 123}, \\
& E I_{i 1} I_{j 2} I_{k 3} I_{\ell 4}=\delta_{i \ldots \ell} D_{i 1234}+\sum^{3} \delta_{i j, k \ell} D_{i 12} D_{k 34}
\end{aligned}
$$

for

$$
\begin{aligned}
\sum^{3} \delta_{i j, k \ell} D_{i 12} D_{k 34}= & \delta_{i j, k \ell} D_{i 12} D_{k 34}+\delta_{i k, j \ell} D_{i 13} D_{j 24}+\delta_{i \ell, j k} D_{i 14} D_{j 23}, \\
E I_{i 1} \ldots I_{m 5}= & \delta_{i \ldots m} D_{i 1 \ldots 5}+\sum^{10} \delta_{i j, k \ell m} D_{i 12} D_{k 345}, \\
E I_{i 1} \ldots I_{n 6}= & \delta_{i \ldots n} D_{i 1 \ldots 6}+\sum^{15} \delta_{i j, k \ldots n} D_{i 12} D_{k 3456} \\
& +\sum^{10} \delta_{i j k, \ell m n} D_{i 123} D_{\ell 456}+\sum^{15} \delta_{i j, k \ell, m n} D_{i 12} D_{k 34} D_{m 56}
\end{aligned}
$$

and $\sum^{10}$ and $\sum^{15}$ defined similarly. Setting $F_{i r}=F_{i}\left(x_{r}\right)$ and $F_{i 1 \wedge 2 \wedge \ldots}=F_{i}\left(\min \left(x_{1}, x_{2}, \ldots\right)\right)$, we have

$$
\begin{aligned}
& D_{i 1}=0 \\
& D_{i 12}=F_{i 1 \wedge 2}-F_{i 1} F_{i 2} \\
& D_{i 123}=F_{i 1 \wedge 2 \wedge 3}-\sum_{123}^{3} F_{i 1} F_{i 2 \wedge 3}+2 F_{i 1} F_{i 2} F_{i 3}, \\
& D_{i 1 \ldots 4}=F_{i 1 \wedge \ldots \wedge 4}-\sum_{1234}^{4} F_{i 1} F_{i 2 \wedge 3 \wedge 4}+\sum_{1234}^{6} F_{i 1} F_{i 2} F_{i 3 \wedge 4}-3 F_{i 1} \ldots F_{i 4},
\end{aligned}
$$


and so on, where $\sum_{1 \ldots r}^{m} g_{1 \ldots r}=\sum g_{\pi}$ summed over all $m$ permutations of $1 \ldots r$ giving distinct terms. So, writing $T_{1 \ldots r}=T_{x_{1} \ldots x_{r}}$,

$$
\begin{aligned}
\alpha_{1} & =E T_{(1)}=n^{-1} \sum_{i} w_{i} \int T_{1} d D_{i 1}=0, \\
\alpha_{2} & =E T_{(2)}=n^{-2} \sum_{i j} w_{i} w_{j} \iint T_{12} d E I_{i 1} I_{j 2} \\
& =n^{-2} \sum_{i} w_{i}^{2} \iint T_{12} d D_{i 12} \\
& =n^{-2} \sum_{i} w_{i}^{2}\left\{[11]_{i}-[12]_{i i}\right\}=n^{-1}\{11\}, \\
\alpha_{3} & =E T_{(3)}=n^{-3} \sum_{i j k} w_{i} w_{j} w_{k} \iiint T_{123} d E I_{i 1} I_{j 2} I_{k 3} \\
& =n^{-3} \sum_{i} w_{i}^{3} \iint T_{123} d D_{i 123}=n^{-2}\{111\}, \\
\alpha_{4} & =E T_{(4)}=n^{-4} \sum_{i \ldots \ell} w_{i} \ldots w_{\ell} \int \ldots \int d E I_{i 1} \ldots I_{\ell 4} \\
& =n^{-3} \alpha_{4 \cdot 1}+3 n^{-2} \alpha_{4 \cdot 2}
\end{aligned}
$$

for

$$
\alpha_{4 \cdot 1}=n^{-1} \sum_{i} w_{i}^{4} \int \ldots \int T_{1 \ldots 4} d D_{i 1 \ldots 4}=O(1)
$$

and

$$
\alpha_{4 \cdot 2}=n^{-2} \sum_{i k}^{\prime} w_{i}^{2} w_{k}^{2} \int \ldots \int T_{1 \ldots 4} d D_{i 12} d D_{k 34},
$$

where $\sum_{i j \ldots}^{\prime}$ sums over distinct $i, j \ldots$ in $1, \ldots, n$. So, $\alpha_{4.2}=\alpha_{4.2}^{\prime}+O\left(n^{-1}\right)$, where $\alpha_{4.2}^{\prime}$ replaces $\sum_{i k}^{\prime}$ by $\sum_{i k}$. So, $\alpha_{4.2}^{\prime}=\{1122\}$. The expressions for $a_{10}, a_{11}, a_{12}$ follow since $a_{1 i}^{\prime}=a_{1 i}$. Also

$$
E\{T(\widehat{F})-T(F)\}^{2}=\alpha_{11}+\alpha_{12}+\alpha_{13} / 3+\alpha_{22} / 4+O\left(n^{-3}\right),
$$

where

$$
\begin{aligned}
\alpha_{11} & =n^{-2} \sum_{i j} w_{i} w_{j} \iint T_{1} T_{2} d E I_{i 1} I_{j 2}=n^{-2} \sum_{i} w_{i}^{2} \iint T_{1} T_{2} d D_{i 12} \\
& =n^{-1}\left\{1^{2}\right\} \\
\alpha_{12} & =n^{-3} \sum_{i j k} w_{i} w_{j} w_{k} \iiint T_{1} T_{23} d E I_{i 1} I_{j 2} I_{k 3} \\
& =n^{-3} \sum_{i} w_{i}^{3} \iiint T_{1} T_{23} d D_{i 123}=n^{-2}\{1,11\}, \\
\alpha_{13} & =n^{-4} \sum_{i \ldots \ell} w_{i} \ldots w_{\ell} \int \ldots \int T_{1} T_{234} d E I_{i 1} \ldots I_{\ell 4}=\alpha_{13.1}+\alpha_{13.2}, \\
\alpha_{22} & =\alpha_{22.1}+\alpha_{22.2}
\end{aligned}
$$


for

$$
\begin{aligned}
& \alpha_{13.1}=n^{-4} \sum_{i} w_{i}^{4} \int \ldots \int T_{1} T_{234} d D_{i 1234}=O\left(n^{-3}\right) \\
& \alpha_{13.2}=n^{-4} \sum_{i k} w_{i}^{2} w_{k}^{2} \int \ldots \int T_{1} T_{234} d\left(D_{i 12} D_{k 34}\right)=\alpha_{13.2}^{\prime}+O\left(n^{-3}\right) \\
& \alpha_{22.1}=n^{-4} \sum_{i} w_{i}^{4} \int \ldots \int T_{12} T_{34} d D_{i 1 \ldots 4}=O\left(n^{-3}\right) \\
& \alpha_{22.2}=\alpha_{22.21}+2 \alpha_{22.22}+O\left(n^{-3}\right) \\
& \alpha_{22.21}=n^{-4} \sum_{i j} w_{i}^{2} w_{j}^{2} \iint T_{12} T_{34} d\left(D_{i 12} D_{j 34}\right)=n^{-2}\{11\}^{2} \\
& \alpha_{22.22}=n^{-4} \sum_{i j} w_{i}^{2} w_{j}^{2} \iint T_{12} T_{34} d\left(D_{i 13} D_{j 24}\right)=n^{-2}\left\{12^{2}\right\}
\end{aligned}
$$

where $\alpha_{13.2}^{\prime}$ replaces $\sum_{i k}^{\prime}$ by $\sum_{i k}$, so $\alpha_{13.2}^{\prime}=n^{-2}\{1,122\}$. So, $a_{21}^{\prime}=\left\{1^{2}\right\}$ and $a_{22}^{\prime}=$ $\{1,11\}+\{1,122\}+\{11\}^{2} / 4+\left\{12^{2}\right\} / 2$. Now use $a_{21}=a_{21}^{\prime}$ and $a_{22}=a_{22}^{\prime}-a_{11}^{2}$. Also

$$
E\{T(\widehat{F})-T(F)\}^{3}=\alpha_{111}+3 \alpha_{112} / 2+O\left(n^{-3}\right),
$$

where

$$
\begin{aligned}
\alpha_{111} & =n^{-3} \sum_{i j k} w_{i} w_{j} w_{k} \iiint T_{1} T_{2} T_{3} d E I_{i 1} I_{j 2} I_{k 3} \\
& =n^{-3} \sum_{i} w_{i}^{3} \iiint T_{1} T_{2} T_{3} d D_{i 123}=n^{-2}\left\{1^{3}\right\}
\end{aligned}
$$

and

$$
\begin{aligned}
\alpha_{112} & =n^{-4} \sum_{i \ldots \ell} w_{i} \ldots w_{\ell} \int \ldots \int T_{1} T_{2} T_{34} d E I_{i 1} \ldots I_{\ell 4} \\
& =\alpha_{112.1}+\alpha_{112.2}+2 \alpha_{112.3}
\end{aligned}
$$

for

$$
\begin{aligned}
\alpha_{112.1} & =n^{-4} \sum_{i} w_{i}^{4} \int \ldots \int T_{1} T_{2} T_{3} d D_{i 1 \ldots 4}=O\left(n^{-3}\right), \\
\alpha_{112.2} & =n^{-4} \sum_{i j}^{\prime} w_{i}^{2} w_{j}^{2} \int \ldots \int T_{1} T_{2} T_{34} d\left(D_{i 12} D_{j 34}\right) \\
& =n^{-2}\left\{1^{2}\right\}\{11\}+O\left(n^{-3}\right), \\
\alpha_{112.3} & =n^{-4} \sum_{i j}^{\prime} w_{i}^{2} w_{j}^{2} \int \ldots \int T_{1} T_{2} T_{34} d\left(D_{i 13} D_{j 24}\right) \\
& =n^{-2}\{1,2,12\}+O\left(n^{-3}\right) .
\end{aligned}
$$


So, $a_{32}^{\prime}=\left\{1^{3}\right\}+3\left\{1^{2}\right\}\{11\} / 2+3\{1,2,12\}$. Now use $a_{32}=a_{32}^{\prime}-3 a_{21} a_{11}$ to obtain $a_{32}$ above. Also

$$
\begin{aligned}
& E\{T(\widehat{F})-T(F)\}^{4}=\alpha_{1111}+2 \alpha_{1112}+2 \alpha_{1113} / 3+3 \alpha_{1122} / 2+O\left(n^{-4}\right), \\
& \alpha_{1111}=\alpha_{1111.1}+3 \alpha_{1111.2}, \\
& \alpha_{1112}=\alpha_{1112.1}+3 \alpha_{1112.2}+6 \alpha_{1112.3}+\alpha_{1112.4}, \\
& \alpha_{1113}=\alpha_{1113.1}+O\left(n^{-4}\right), \\
& \alpha_{1122}=\alpha_{1122.1}+O\left(n^{-4}\right)
\end{aligned}
$$

for

$$
\begin{aligned}
& \alpha_{1111.1}=n^{-4} \sum_{i} w_{i}^{4} \int \ldots \int T_{1} T_{2} T_{3} T_{4} d D_{1234}=n^{-3}\left\{1^{4}\right\}, \\
& \alpha_{1111.2}=n^{-4} \sum_{i j}^{\prime} w_{i}^{2} w_{j}^{2} \int \ldots \int T_{1} \ldots T_{4} d\left(D_{i 12} D_{j 34}\right) \\
& =n^{-2}\left\{1^{2}\right\}^{2}-n^{-3}\left\{1^{2}, 1^{2}\right\} \text {, } \\
& \alpha_{1112.1}=n^{-5} \sum_{i} w_{i}^{5} \int \ldots \int T_{1} T_{2} T_{3} T_{45} d D_{i 1 \ldots 5}=O\left(n^{-4}\right) \text {, } \\
& \alpha_{1112.2}=n^{-5} \sum_{i j}^{\prime} w_{i}^{2} w_{j}^{3} \int \ldots \int T_{1} T_{2} T_{3} T_{45} d\left(D_{i 12} D_{j 345}\right) \\
& =n^{-3}\left\{1^{2}\right\}\{1,11\}+O\left(n^{-4}\right), \\
& \alpha_{1112.3}=n^{-5} \sum_{i j}^{\prime} w_{i}^{2} w_{j}^{3} \int \ldots \int T_{1} T_{2} T_{3} T_{45} d\left(D_{i 14} D_{j 235}\right) \\
& =n^{-3}\left\{1,12,2^{2}\right\}+O\left(n^{-4}\right) \text {, } \\
& \alpha_{1112.4}=n^{-5} \sum_{i j}^{\prime} w_{i}^{2} w_{j}^{3} \int \ldots \int T_{1} T_{2} T_{3} T_{45} d\left(D_{i 45} D_{j 123}\right) \\
& =n^{-3}\left\{1^{3}\right\}\{11\}+O\left(n^{-4}\right) \text {, } \\
& \alpha_{1113.1}=n^{-6} \sum_{i j k} w_{i}^{2} w_{j}^{2} w_{k}^{2} \int \ldots \int T_{1} T_{2} T_{3} T_{456} d \sum^{15} D_{i 12} D_{j 34} D_{k 56} \\
& =9 \alpha_{1113.2}+6 \alpha_{1113.3} \text {, } \\
& \alpha_{1113.2}=n^{-6} \sum_{i j k} w_{i}^{2} w_{j}^{2} w_{k}^{2} \int \ldots \int T_{1} T_{2} T_{3} T_{456} d\left(D_{i 12} D_{j 34} D_{k 56}\right) \\
& =n^{-3}\left\{1^{2}\right\}\{1,122\} \text {, } \\
& \alpha_{1113.3}=n^{-6} \sum_{i j k} w_{i}^{2} w_{j}^{2} w_{k}^{2} \int \ldots \int T_{1} T_{2} T_{3} T_{456} d\left(D_{i 14} D_{j 25} D_{k 36}\right) \\
& =n^{-3}\{1,2,3,123\} \text {, } \\
& \alpha_{1122.1}=n^{-6} \sum_{i j k} w_{i}^{2} w_{j}^{2} w_{k}^{2} \int \ldots \int T_{1} T_{2} T_{34} T_{56} d \sum^{15} D_{i 12} D_{j 34} D_{k 56} \\
& =n^{-3}\left(\gamma_{1}+4 \gamma_{2}+8 \gamma_{3}+2 \gamma_{4}\right) \text {, }
\end{aligned}
$$


where

$$
\begin{aligned}
\gamma_{1} & =n^{-3} \sum_{i j k} w_{i}^{2} w_{j}^{2} w_{k}^{2} \int \ldots \int T_{1} T_{2} T_{34} T_{56} d\left(D_{i 12} D_{j 34} D_{k 56}\right) \\
& =\left\{1^{2}\right\}\{11\}^{2}, \\
\gamma_{2} & =n^{-3} \sum_{i j k} w_{i}^{2} w_{j}^{2} w_{k}^{2} \int \ldots \int T_{1} T_{2} T_{34} T_{56} d\left(D_{i 13} D_{j 24} D_{k 56}\right) \\
& =\{11\}\{1,2,12\}, \\
\gamma_{3} & =n^{-3} \sum_{i j k} w_{i}^{2} w_{j}^{2} w_{k}^{2} \int \ldots \int T_{1} T_{2} T_{34} T_{56} d\left(D_{i 13} D_{j 45} D_{k 26}\right) \\
& =\{1,13,32,2\}, \\
\gamma_{4} & =n^{-3} \sum_{i j k} w_{i}^{2} w_{j}^{2} w_{k}^{2} \int \ldots \int T_{1} T_{2} T_{34} T_{56} d\left(D_{i 12} D_{j 35} D_{k 46}\right) \\
& =\left\{1^{2}\right\}\left\{12^{2}\right\} .
\end{aligned}
$$

So, $a_{42}^{\prime}=3\left\{1^{2}\right\}^{2}$ and $a_{43}^{\prime}=a_{43}+4 a_{11} a_{32}+6 a_{21} a_{22}^{\prime}$ for $a_{43}$ as above. Now use $a_{43}=$ $a_{43}^{\prime}-4 a_{11} a_{32}-6 a_{21} a_{22}^{\prime}$.

Note B.1 An alternative method better for obtaining these expressions for the cumulant coefficients $a_{r i}$, is to use the parametric approach of Withers (1988). This is possible since

$$
\kappa\left(\widehat{F}\left(x_{1}\right), \ldots, \widehat{F}\left(x_{r}\right)\right)=n^{1-r} k\left(x_{1} \ldots x_{r}\right),
$$

where

$$
k\left(x_{1} \ldots x_{r}\right)=n^{-1} \sum_{i=1}^{r} w_{i}^{r} k\left(x_{1} \ldots x_{r} F_{i}\right)
$$

and $k\left(x_{1} \ldots x_{r} F_{i}\right)=\kappa\left(I\left(X_{i} \leq x_{1}\right), \ldots, I\left(X_{i} \leq x_{r}\right)\right)$. 